\title{
Globular cluster clustering around ultra compact dwarf galaxies in the halo of NGC 1399
}

\author{
Karina Voggel ${ }^{1}$, Michael Hilker ${ }^{1}$ and Tom Richtler ${ }^{2}$ \\ ${ }^{1}$ European Southern Observatory, Garching, Germany, email: kvoggel@eso.org \\ ${ }^{2}$ Universidad de Concepción, Concepción, Chile
}

\begin{abstract}
We tested the spatial distribution of UCDs and GCs in the halo of NGC 1399 in the Fornax cluster. In particular we tried to find out if globular clusters are more abundant in the vicinity of UCDs than what is expected from their global distribution. A local overabundance of globular clusters was found around UCDs on a scale of $1 \mathrm{kpc}$ compared to what is expected from the large scale distribution of globulars in the host galaxy. This effect is stronger for the metal-poor blue GCs and weaker for the red GCs. An explanation for these clustered globulars is either that they are the remains of a GC system of an ancestor dwarf galaxy before it was stripped to its nucleus, which appears as UCD today. Alternatively these clustered GCs could have been originally part of a super star cluster complex.
\end{abstract}

Keywords. galaxies: clusters: individual: Fornax, galaxies: dwarf, galaxies: star clusters

\section{Introduction and method}

The first Ultra-Compact Dwarf Galaxies (UCDs) were detected in the Fornax cluster (Hilker et al. 1999). Their discovery filled the empty gap in size and magnitude between globular clusters (GCs) and galaxies that was not populated before. The formation and origin of these objects is still unclear. Three main ways of forming an UCD are discussed: 1.) They are the stripped remnant nuclei of a larger dwarf galaxy (Bekki al. 2003), 2.) they are from the high mass extension of the GC mass function (Murray et al. 2009) or 3.) they are the remnants of a super star cluster complexes that merged together and formed the UCD (Fellhauer \& Kroupa 2002).

We are using the catalogue of globular clusters around NGC 1399 provided by Dirsch et al. (2003) to study their spatial distribution around UCDs. We derived the radial surface density distribution of GCs in the halo. The resulting power-law distribution is then assumed to be the expected value of GCs at each radial distance to NGC 1399 . Then the local GC density around UCDs is computed and compared to the expected GC density from a global GC distribution.

\section{Results and conclusion}

The derived density ratio between expected and measured density, is shown in the left panel of figure 1. This clustering signal is the average for all 206 UCDs (green triangles). The same analysis was done for red and blue GCs only, which is shown in its respective colors. In the right panel the same figure is shown for the subset of 100 UCDs that have a GC candidate within $1 \mathrm{kpc}$ to emphasize the difference in clustering between this population and the comparison GC population plotted in black. We find an increasing GC density around UCDs at scales smaller than $1 \mathrm{kpc}$ for the full sample, which is distinguished from the GCs in their clustering properties. In the right panel of fig. 1 this 

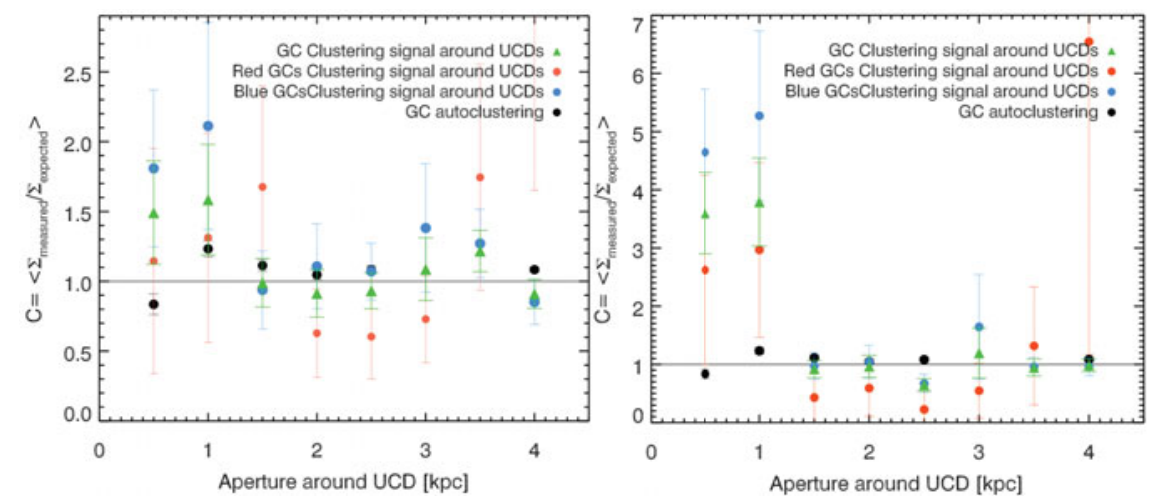

Figure 1. Left panel: clustering signal of GCs around UCDs at radial distances from the UCDs for the full GC population (green), and the red and blue populations. Black data points are the autoclustering of GCs. At each radial distance the clustering signal is the average for the 206 UCDs. Right panel: Same plot for 100 UCDs, which have GC candidate within $r<1 \mathrm{kpc}$.

difference is more pronounced, when selecting only a subset of UCDs. When testing the clustering of blue and red GCs separately we find that the clustering shows the same type of signal, but the association of metal-poor blue GCs with the UCDs is stronger than for the red ones.

One explanation for the clustering of GCs in the vicinity of UCDs could be that UCDs are the nuclei of an ancestor dwarf galaxy that possessed its own GC system. The detected clustering would then constitute the remains of the GC population of the initial galaxy before it was affected by stripping. This GC clustering is also expected in the case when globular clusters merge towards the center of their host galaxy via dynamical friction. As the observed clustering is strongest for the blue GC subpopulation, this supports the view that some UCDs with blue companions are the stripped nuclei of metal-poor (dwarf) galaxies.

A second explanation for the clustering of GCs around UCDs could be that these UCDs were formed in a large star cluster complex. This complex then merged to form the UCD (Fellhauer \& Kroupa 2002). The observed overdensites of star clusters around UCDs could then be the remains of this process before a fully merged object is created. The simulations of Brüns et al. (2011) predict that these large star cluster complexes have typical merging timescales of $1 \mathrm{Gyr}$. In some cases though, GC substructure around the central object is still detectable after 5 Gyrs. Thus, if a UCD and its surrounding GCs are formed by super star clusters, we expect young ages and high metallicities.

We conclude that UCDs can be distinguished into two different populations: 1) UCDs that harbour a population of close-by satellite point sources, most probably low mass star clusters; 2) UCDs that have the same statistical clustering properties as 'normal' globular clusters. Further details on this work can be found in Voggel, Hilker \& Richtler (2015).

\section{References}

Bekki, K., Couch, W. J., Drinkwater, M. J., \& Shioya, Y., 2003, MNRAS, 344,399

Brüns, C., Kroupa, P., Fellhauer, M., Metz, M., \& Assmann, P. 2011, A\&\&A, 529, A138

Dirsch, B., Richtler, T., Geisler, D., et al., 2003, AJ, 125, 1908

Fellhauer, M., \& Kroupa, P., 2002, MNRAS, 330, 642

Hilker, M., Infante, L., Vieira, G., Kissler-Patig, M., \& Richtler, T., 1999, A\& $A S$, 134, 75

Murray, N., 2009, ApJ, 691, 946

Voggel, K., Hilker, M., \& Richtler, T. 2015, submitted to A\&SA 